\title{
Robust L2 Disturbance Attenuation for A Class of Uncertain Lipschitz Nonlinear Systems with Input Delay
}

DOI:

10.1080/00207179.2017.1378442

\section{Document Version}

Accepted author manuscript

Link to publication record in Manchester Research Explorer

\section{Citation for published version (APA):}

Zuo, Z., Wang, C., Wen, Y., \& Ding, Z. (2017). Robust $L_{2}$ Disturbance Attenuation for A Class of Uncertain Lipschitz Nonlinear Systems with Input Delay. International Journal of Control.

https://doi.org/10.1080/00207179.2017.1378442

\section{Published in:}

International Journal of Control

\section{Citing this paper}

Please note that where the full-text provided on Manchester Research Explorer is the Author Accepted Manuscript or Proof version this may differ from the final Published version. If citing, it is advised that you check and use the publisher's definitive version.

\section{General rights}

Copyright and moral rights for the publications made accessible in the Research Explorer are retained by the authors and/or other copyright owners and it is a condition of accessing publications that users recognise and abide by the legal requirements associated with these rights.

\section{Takedown policy}

If you believe that this document breaches copyright please refer to the University of Manchester's Takedown Procedures [http://man.ac.uk/04Y6Bo] or contact uml.scholarlycommunications@manchester.ac.uk providing relevant details, so we can investigate your claim.

\section{OPEN ACCESS}




\title{
ARTICLE
}

\section{Robust $\mathcal{L}_{2}$ Disturbance Attenuation for A Class of Uncertain Lipschitz Nonlinear Systems with Input Delay}

\author{
Zongyu Zuo ${ }^{\mathrm{a}}$, Chunyan Wang ${ }^{\mathrm{b}}$, Wen Yang ${ }^{\mathrm{c}}$ and Zhengtao Ding ${ }^{\mathrm{b}}$ \\ aThe Seventh Research Division, and Science and Technology on Aircraft Control \\ Laboratory, Beihang University (BUAA), Beijing 100191, China \\ ${ }^{\mathrm{b}}$ School of Electrical and Electronic Engineering, University of Manchester, Sackville Street \\ Building, Manchester M13 9PL, UK \\ ${ }^{\mathrm{c}}$ Key Laboratory of Advanced Control and Optimization for Chemical Processes, East China \\ University of Science and Technology, Shanghai 200237, China
}

\begin{abstract}
ARTICLE HISTORY
Compiled September 6, 2017

ABSTRACT

In this paper, we study a robust $\mathcal{L}_{2}$ disturbance attenuation problem that arises when applying the Artstein-Kwon-Pearson reduction transformation for a class of uncertain Lipschitz nonlinear systems with input delay and external disturbances. A conventional predictor-based feedback controller is adopted with the control gain matrix carefully identified by solving a couple of sufficient conditions in terms of Linear Matrix Inequalities (LMIs). Lyapunov-Krasovskii functionals are constructed to guarantee that the robust $\mathcal{L}_{2}$ disturbance attenuation problem can be solved by the proposed controller. A numerical example is included to validate the performance of the proposed controller.
\end{abstract}

\section{KEYWORDS}

Disturbance attenuation; input delay; Lipschitz nonlinearity; parametric uncertainty; robust stabilization

\section{Introduction}

Time delays widely exist in many industrial processes due to the time taken for transmission of signals, transport of materials, etc. For the analysis and control of aircraft turbofan engine as an example, pure time delays must be included during the process of modeling and may vary as a function of fan speed, altitude and Mach number (Hueschen, 2011). The presence of time delays, if not considered in control design, may lead to the significant performance degradation of the controlled system. For this reason, the research on delay systems in control engineering has been active for a long time (Richard, 2003).

It is known from the perspective of control theory that stabilization of input delayed systems are more involved than the state delayed systems counterpart. One basic idea in tackling input delay is to predict the evolution of the state variable for the delay period and then use the predicted state for control. The Smith predictor (Smith, 1959) is an early result for stable linear systems in frequency domain, which has been widely 
used in industrial applications. For general (possible unstable) linear systems in the time domain, the reduction method (Artstein, 1982; Kwon \& Pearson, 1980), also known as finite spectrum assignment technique (Manitius \& Olbrot, 1979; Mondie \& Michiels, 2003), is one of the most popular approaches to cope with delays in the input, which uses a state predictor with an integral operator to transform the original system to a delay-free one for control design. Recently, the prediction method is extended to several specific classes of nonlinear systems (e.g., nonlinear strict-feedback systems (Krstic, 2010)) with more recent results in (Bekiaris-Liberis \& Krstic, 2012, 2013; Mazenc et al., 2012; Pasillas-Lépine et al., 2013). For conventional predictor-based methods, difficulties arise in computation of the prediction term involving on-line integration. The truncated prediction feedback (TPF) control (Lin \& Fang, 2007) is developed with the integral part dropped for both general linear systems (Yoon \& Lin, 2013; Zhou et al., 2010, 2012) and Lipschitz nonlinear systems (Zuo et al., 2016).

However, the predictor-based feedback control methods suffer from a difficulty in practical implementation when uncertainties exist in system dynamics. The TPF control also encounters the same barrier since the prediction is based on the exponential of the exact system matrix. In real-world scenarios, it may induce unstable behavior when applying the existing predictor feedback controller without considering the system uncertainties. For linear input-delayed systems with parametric uncertainties, LQR control (Choi \& Chung, 1995) and robust control (Kim et al., 1996) have been proposed to guarantee the delay-independent stability of the closed-loop systems. As pointed out in (Moon et al., 2001), the derived memoryless controllers are very conservative especially when the actual delay is small. To overcome this conservativeness, the reduction transformation is applied in (Moon et al., 2001) to the uncertain inputdelayed systems and a robust stabilizing controller is constructed by solving convex optimization problems. But for uncertain nonlinear input-delayed systems, very a few results are reported till now. Taking into account the nonlinear parameter perturbations, the work (Roh \& Oh, 1999) applied the reduction transformation to compensate for the input delay and then proposed a sliding mode control to eliminate the nonlinear perturbations. Unfortunately, the nonlinear function are very restrictive to be matched uncertainties (Nguang, 2001).

Motivated by the existing results, this paper considers the robust control problem for input-delayed nonlinear systems with both parametric uncertainties and external disturbances. In addition, the nonlinear function considered in the system dynamics is restrictive to be Lipschitz as a perturbation. In viewing the possible heavy dependence on the precise system dynamics of TPF method, classical reduction transformation is applied to deal with the input delay. However, the transformed system is no longer delay free due to the parametric uncertainties and the nonlinear functions. In particular, the nonlinearity is still a function of the original state after transformation, which poses a challenge to control design and stability analysis. To guarantee the closed-loop stability and attenuate the unknown external disturbances, a set of conditions based on Lyapunov-Krasovskii analysis for the robust $\mathcal{L}_{2}$ disturbance attenuation problem are established. Finally, a numerical example is introduced to show the design procedure proposed in this paper.

This paper is organized as follows. The well-known reduction method and a lemma are recalled in Section 2. Section 3 formulates the disturbance attenuation problem to be solved. In Section 4, sufficient conditions are established with rigorous stability analysis. Section 5 introduces a control design with simulation results as an example to illustrate the proposed design. Finally, the paper ends concluding remarks in Section 6. 


\section{Preliminaries}

In this section, we recall briefly the Artstein-Kwon-Pearson reduction method (Kwon \& Pearson, 1980) for general linear input-delayed systems

$$
\dot{x}(t)=A x(t)+B u(t-h),
$$

where $x \in \mathbb{R}^{n}$ is the state, $u \in \mathbb{R}^{p}$ is the input, $h \in \mathbb{R}_{+}$is the input delay, $A \in$ $\mathbb{R}^{n \times n}, B \in \mathbb{R}^{n \times p}$ are constant matrices with $(A, B)$ being controllable, and the initial conditions $x(\theta), \theta \in[-h, 0]$, are bounded.

Introduce a reduction transformation

$$
z(t)=x(t)+\int_{t}^{t+h} \mathrm{e}^{A(t-\tau)} B u(\tau-h) \mathrm{d} \tau
$$

Differentiating $z(t)$ against time, we have

$$
\begin{aligned}
\dot{z}(t) & =A x(t)+\mathrm{e}^{-A h} B u(t)+A \int_{t}^{t+h} \mathrm{e}^{A(t-\tau)} B u(\tau-h) \mathrm{d} \tau \\
& =A z(t)+D u(t)
\end{aligned}
$$

where $D=\mathrm{e}^{-A h} B$. Thus, the original system (1) is transformed to a delay-free one (3) via the transformation (2). Note that the controllability of $(A, B)$ and $\left(A, e^{-A h} B\right)$ are equivalent.

Let's consider a controller

$$
u(t)=K z(t) .
$$

where $K$ is the control gain matrix. Then, we have from (2) and (4) that

$$
\|x(t)\| \leq\|z(t)\|+h\left(\max _{-h \leq \theta \leq 0}\left\|e^{A \theta}\right\|\right)\|B\|\|K\|\left\|z_{t}(\theta)\right\|,
$$

where $z_{t}(\theta):=z(t+\theta),-h \leq \theta \leq 0$, which implies that $x(t)$ approaches 0 as $z(t)$ approaches 0 . In other words, if the controller (4) stabilizes the transformed system (3), then the original system (1) subject to the same controller is also stable (Kwon \& Pearson, 1980).

Finally, we recall a lemma (Gu, 2010) for stability analysis.

Lemma 2.1. For a positive definite matrix $P$, and a function $x:[a, b] \rightarrow \mathbb{R}^{n}$, with $a, b \in \mathbb{R}$ and $b>a$, the following inequality holds:

$$
\begin{aligned}
\left(\int_{a}^{b} x^{T}(\tau) d \tau\right) & P\left(\int_{a}^{b} x(\tau) d \tau\right) \\
\leq & (b-a) \int_{a}^{b} x^{T}(\tau) P x(\tau) d \tau
\end{aligned}
$$




\section{Problem Statement}

In this paper, we consider robust stabilization problem for a class of uncertain Lipschitz nonlinear systems with input delay and external disturbances, given by

$$
\begin{aligned}
\dot{x}(t)= & (A+\triangle A(t)) x(t)+(B+\triangle B(t)) u(t-h) \\
& +\phi(x(t))+B_{1} \omega(t),
\end{aligned}
$$

where $x \in \mathbb{R}^{n}$ is the state, $u \in \mathbb{R}^{p}$ is the input, $h \in \mathbb{R}_{+}$is the input delay, $A \in$ $\mathbb{R}^{n \times n}, B \in \mathbb{R}^{n \times p}$ and $B_{1} \in \mathbb{R}^{n \times m}$ are nominal constant matrices with $(A, B)$ being controllable, the initial conditions $x(\theta), \theta \in[-h, 0]$, are bounded, $\triangle A(t)$ and $\triangle B(t)$ are time-varying uncertain matrices, $\omega(t) \in \mathcal{L}_{2}^{m}[0, \infty)$ are unknown external disturbances, and $\phi: \mathbb{R}^{n} \rightarrow \mathbb{R}^{n}, \phi(0)=0$, is an unknown global Lipschitz nonlinear function with a Lipschitz constant $\gamma$. For any two constant vectors $a, b \in \mathbb{R}^{n}$,

$$
\|\phi(a)-\phi(b)\| \leq \gamma\|a-b\| .
$$

For system (6), the following two assumptions are made to facilitate the robust control design in the sequel.

Assumption 3.1. The Lipschitz constant $\gamma$ in (7) is a priori known for the nonlinear function $\phi(x(t))$.

Assumption 3.2. The parametric uncertainties $\triangle A(t)$ and $\triangle B(t)$ are assumed to be norm bounded in the form

$$
\Delta A(t)=E \Sigma(t) F_{1} \quad \text { and } \quad \Delta B(t)=E \Sigma(t) F_{2},
$$

where $E, F_{1}$ and $F_{2}$ are real constant matrices with appropriate dimensions, and $\Sigma(t)$ is an unknown real time-varying matrix with Lebesgue-measurable elements satisfying $\Sigma^{T}(t) \Sigma(t) \leq I$.

Remark 1. The nonlinearities in (6) are restricted to being Lipschitz, say Assumption 3.1, which allows less restriction on the structure of nonlinearity and may result from the un-modeled dynamics or the linearization errors around operating points. Assumption 3.2 is very essential for robust control design, which allows the parameter perturbations to be time-varying. Thus, our formulation in this paper covers a wide class of systems.

Following the reduction method, we consider the same transformation (2) for the system in (6). In this case, it can be transformed to

$$
\begin{aligned}
\dot{z}(t)= & (A+\triangle A(t)) z(t)+D u(t)+\triangle B(t) u(t-h)+\phi(x(t)) \\
& +B_{1} \omega(t)-\triangle A(t) \int_{t}^{t+h} e^{A(t-\tau)} B u(\tau-h) \mathrm{d} \tau,
\end{aligned}
$$

where $D=\mathrm{e}^{-A h} B$. It is worth noting from (9) that system (6) is not completely reduced to a delay-free system due to the existence of the parametric uncertainties and the Lipschitz nonlinearity.

Employing the same control structure as in (4) for (9), we specify the control gain 
matrix as

$$
K=-D^{T} P
$$

where $P>0$ is a positive definite matrix to be designed later. The gain matrix $K$ in (10) is actually a classic LQ (Linear Quadratic) optimal control gain (Anderson \& Moore, 1989) with $R=I$.

Definition 3.3. The robust $\mathcal{L}_{2}$ disturbance attenuation problem of the nonlinear system (6) is said to be solved by the control input (4) if the following items are satisfied:

1) The uncertain closed-loop system (6) with $\omega(t) \equiv 0$ are asymptotically stable at the origin.

2) For any $t \geq t_{0}$ and $t_{0} \geq 0$, there exists a scalar $\kappa_{1}$ such that

$$
\int_{t_{0}}^{t}\|x(\tau)\|^{2} \mathrm{~d} \tau \leq \bar{\alpha}\left(t_{0}\right)+\frac{1}{\kappa_{1}} \int_{t_{0}}^{t} \bar{\beta}(\|\omega(\tau)\|) \mathrm{d} \tau,
$$

where $\bar{\alpha}\left(t_{0}\right)$ is a positive constant depending on the initial conditions at time $t_{0}$ and $\bar{\beta}(\cdot)$ is a positive definite gain function.

In other words, the control design problem in this paper is to find possible positive definite matrices $P$ such that the robust $\mathcal{L}_{2}$ disturbance attenuation problem is solved.

\section{Main Results}

This section establishes sufficient conditions for the positive definite matrix $P$ in (10) such that the predictor-based control (4) stabilizes the system (9).

Under the control input (4) with (10), the closed-loop system (9) can be written as

$$
\begin{aligned}
\dot{z}(t)= & \left(A-D D^{T} P\right) z(t)+\triangle A z(t)-\triangle B D^{T} P z(t-h) \\
& +\triangle A \int_{t}^{t+h} e^{A(t-\tau)} B D^{T} P z(\tau-h) \mathrm{d} \tau \\
& +\phi(x)+B_{1} \omega(t)
\end{aligned}
$$

Note from (12) that the nonlinear term $\phi(x)$ in the transformed system is still expressed as a function of the original state $x$. However, for the stability analysis, we need to establish a bound of the nonlinear function in terms of the new state $z$.

The following lemma prescribe a bound for the nonlinear function with respect to $z$.

Lemma 4.1. For the Lipschitz nonlinear function $\phi(x)$ in (12), a bound can be established in terms of the state $z$ as follows:

$$
\begin{gathered}
\|\phi(x)\|^{2} \leq \gamma^{2} 2\|z(t)\|^{2}+2 h \gamma^{2} \int_{t-h}^{t} z^{T}(\tau) P D B^{T} e^{A^{T}(t-\tau-h)} \\
\times e^{A(t-\tau-h)} B D^{T} P z(\tau) \mathrm{d} \tau
\end{gathered}
$$

where $\gamma$ is the Lipschitz constant defined in (7). 
Proof. From Lipschitz condition (7), we have

$$
\phi^{T}(x) \phi(x) \leq \gamma^{2} x^{T}(t) x(t)=\gamma^{2}\|x(t)\|^{2} .
$$

Based on the reduction transformation (2), we have

$$
\begin{aligned}
x(t) & =z(t)-\int_{t}^{t+h} \mathrm{e}^{A(t-\tau)} B u(\tau-h) \mathrm{d} \tau \\
& =z(t)+\int_{t-h}^{t} \mathrm{e}^{A(t-\tau-h)} B D^{T} P z(\tau) \mathrm{d} \tau
\end{aligned}
$$

It follows that

$$
\begin{aligned}
\|x(t)\|^{2} \leq & 2\|z(t)\|^{2}+2\left(\int_{t-h}^{t} \mathrm{e}^{A(t-\tau-h)} B D^{T} P z(\tau) \mathrm{d} \tau\right)^{T} \\
& \times\left(\int_{t-h}^{t} \mathrm{e}^{A(t-\tau-h)} B D^{T} P z(\tau) \mathrm{d} \tau\right) \\
\leq & 2\|z(t)\|^{2}+2 h \int_{t-h}^{t} z^{T}(\tau) P D B^{T} e^{A^{T}(t-\tau-h)} \\
& \times e^{A(t-\tau-h)} B D^{T} P z(\tau) \mathrm{d} \tau,
\end{aligned}
$$

where Lemma 2.1 is employed to derive the last inequality. It is straightforward to derive the bound (13) by substituting (15) into (14). This completes the proof.

The following theorem gives a set of sufficient conditions for $P$ to solve the robust stabilization problem for system (6).

Theorem 4.2. Consider the uncertain Lipschitz nonlinear system (6) with Assumptions 3.1 and 3.2. The predictor-based feedback control (4) with (10) solves the robust $\mathcal{L}_{2}$ disturbance attenuation problem if the following conditions are satisfied, for $X=P^{-1}>0, Y>0$,

$$
\begin{gathered}
{\left[\begin{array}{ccc}
Y & D F_{2}^{T} \\
F_{2} D^{T} & \frac{1}{\mu} I
\end{array}\right]>0,} \\
{\left[\begin{array}{cccc}
U & X F_{1}^{T} & X & D D^{T} \\
F_{1} X & -\frac{1}{\varepsilon} I & 0 & 0 \\
X & 0 & -\frac{1}{\gamma^{2}+\kappa_{1}} I & 0 \\
D^{T} D & 0 & 0 & -\frac{1}{\rho} W
\end{array}\right]<0,}
\end{gathered}
$$

where $\varepsilon>0, \mu>0, \rho>0$ and $\epsilon>0$ are all scalars,

$$
\begin{aligned}
U= & A X+X A^{T}-2 D D^{T}+\left(\frac{1}{\varepsilon}+\frac{1}{\mu}+\frac{1}{\rho}\right) E E \\
& +\frac{2}{\epsilon} I+Y \\
W^{-1} \geq & h \int_{0}^{h} e^{A^{T} s}\left(\rho F_{1}^{T} F_{1}+2 \epsilon \gamma^{2} I\right) e^{A s} \mathrm{~d} s .
\end{aligned}
$$


Proof. Let's first try a Lyapunov function

$$
V_{0}(z(t))=z^{T}(t) P z(t)
$$

With (12), a direct calculation of the time derivative of $V_{0}(z(t))$ gives

$$
\begin{aligned}
\dot{V}_{0}= & z^{T}(t)\left(A^{T} P+P A-2 P D D^{T} P\right) z(t)+2 z^{T}(t) P \triangle A z(t) \\
& -2 z^{T}(t) P \triangle B D^{T} P z(t-h)+2 z^{T}(t) P\left(\phi(x)+B_{1} \omega(t)\right) \\
& +2 z^{T}(t) P \triangle A \int_{t-h}^{t} e^{A(t-\tau-h)} B D^{T} P z(\tau) \mathrm{d} \tau \\
\leq & z^{T}(t)\left[A^{T} P+P A-2 P D D^{T} P+\frac{2}{\epsilon} P P+\varepsilon F_{1}^{T} F_{1}\right. \\
& \left.+\left(\frac{1}{\varepsilon}+\frac{1}{\mu}+\frac{1}{\rho}\right) P E E^{T} P\right] z(t)+\epsilon \phi^{T}(x) \phi(x) \\
& +\epsilon \beta\|\omega(t)\|^{2}+\mu z^{T}(t-h) P D F_{2}^{T} F_{2} D^{T} P z(t-h) \\
& +\rho\left(\int_{t-h}^{t} e^{A(t-\tau-h)} B D^{T} P z(\tau) \mathrm{d} \tau\right)^{T} F_{1}^{T} F_{1} \\
& \times\left(\int_{t-h}^{t} e^{A(t-\tau-h)} B D^{T} P z(\tau) \mathrm{d} \tau\right),
\end{aligned}
$$

where we have used Assumption 3.2 and the inequality

$$
\pm a^{T} b \leq \frac{1}{\varepsilon} a^{T} a+\varepsilon b^{T} b
$$

for any $a, b \in \mathbb{R}^{n}, \varepsilon, \mu, \rho, \epsilon>0, \beta$ is a positive number such that $\beta I \geq B_{1}^{T} B_{1}$. Applying Lemma 2.1 and the bound (13) derived in Lemma 4.1 to (21), we have

$$
\begin{aligned}
\dot{V}_{0} \leq & z^{T}(t)\left[A^{T} P+P A-2 P D D^{T} P+\frac{2}{\epsilon} P P+\varepsilon F_{1}^{T} F_{1}\right. \\
& \left.+\left(\frac{1}{\varepsilon}+\frac{1}{\mu}+\frac{1}{\rho}\right) P E E^{T} P\right] z(t)+\epsilon \phi^{T}(x) \phi(x) \\
& +\epsilon \beta\|\omega(t)\|^{2}+\mu z^{T}(t-h) P D F_{2}^{T} F_{2} D^{T} P z(t-h) \\
& +h \rho \int_{t-h}^{t} z^{T}(\tau) P D B^{T} e^{A^{T}(t-\tau-h)} F_{1}^{T} F_{1} \\
& \quad \times e^{A(t-\tau-h)} B D^{T} P z(\tau) \mathrm{d} \tau \\
\leq & z^{T}(t)\left[A^{T} P+P A-2 P D D^{T} P+\frac{2}{\epsilon} P P+\varepsilon F_{1}^{T} F_{1}\right. \\
& \left.+\left(\frac{1}{\varepsilon}+\frac{1}{\mu}+\frac{1}{\rho}\right) P E E^{T} P+2 \epsilon \gamma^{2} I\right] z(t) \\
& +\epsilon \beta\|\omega(t)\|^{2}+\mu z^{T}(t-h) P D F_{2}^{T} F_{2} D^{T} P z(t-h) \\
& +h \rho \int_{0}^{h} z^{T}(t-s) P D D^{T} e^{A^{T} s}\left(\rho F_{1}^{T} F_{1}+2 \epsilon \gamma^{2} I\right) \\
& \times e^{A s} D D^{T} P z(t-s) \mathrm{d} s,
\end{aligned}
$$


where $D=e^{-A h} B$ and $s=t-\tau$ are inserted in the last inequality.

Next, we consider the following Krasovskii functionals

$$
\begin{aligned}
V_{1}\left(z_{t}\right) & =\int_{t-h}^{t} z^{T}(\tau) R z(\tau) \mathrm{d} \tau \\
V_{2}\left(z_{t}\right)= & h \rho \int_{0}^{h} \int_{t-s}^{t} z^{T}(\tau) P D D^{T} e^{A^{T} s}\left(\rho F_{1}^{T} F_{1}+2 \epsilon \gamma^{2} I\right) \\
& \times e^{A s} D D^{T} P z(\tau) \mathrm{d} \tau \mathrm{d} s,
\end{aligned}
$$

where

$$
R-\mu P D F_{2}^{T} F_{2} D^{T} P>0 .
$$

A direct calculation gives that

$$
\begin{gathered}
\dot{V}_{1}=z^{T}(t) R z(t)-z^{T}(t-h) R z(t-h) \\
\dot{V}_{2}=\rho z^{T}(t) P D D^{T}\left[h \int_{0}^{h} e^{A^{T} s}\left(\rho F_{1}^{T} F_{1}+\epsilon \gamma^{2} I\right) e^{A s} \mathrm{~d} s\right] \\
\times D D^{T} P z(t) \\
-h \rho \int_{0}^{h} z^{T}(t-s) P D D^{T} e^{A^{T} s}\left(\rho F_{1}^{T} F_{1}+\epsilon \gamma^{2} I\right) \\
\times e^{A s} D D^{T} P z(t-s) \mathrm{d} s,
\end{gathered}
$$

Consider the following Lyapunov-Krasovskii functional

$$
V\left(z_{t}\right)=V_{0}(z)+V_{1}\left(z_{t}\right)+V_{2}\left(z_{t}\right)
$$

which is radially unbounded with respect to $z(t)$. Let $W^{-1}$ be a positive definite matrix satisfying (19). A direct evaluation gives that

$$
\begin{aligned}
\dot{V}\left(z_{t}\right) & =\dot{V}_{0}(z)+\dot{V}_{1}\left(z_{t}\right)+\dot{V}_{2}\left(z_{t}\right) \\
& \leq z^{T}(t) Q z(t)+\bar{\beta}(\|\omega(t)\|),
\end{aligned}
$$

where

$$
\begin{aligned}
Q= & A^{T} P+P A-2 P D D^{T} P+\left(\frac{1}{\varepsilon}+\frac{1}{\mu}+\frac{1}{\rho}\right) P E E^{T} P \\
& +\frac{2}{\epsilon} P P+\varepsilon F_{1}^{T} F_{1}+\rho P D D^{T} W^{-1} D D^{T} P \\
& +R+2 \epsilon \gamma^{2} I,
\end{aligned}
$$

and

$$
\bar{\beta}(\|\omega(t)\|)=\epsilon \beta\|\omega(t)\|^{2} .
$$


By Lyapunov-Krasovskii Stability Theorem (Gu et al., 2003), global asymptotical stability can be established if the inequalities (19), (25) and $Q<0$ hold. It is easy to show that inequality (25) can be written as

$$
P^{-1} R P^{-1}-\mu D F_{2}^{T} F_{2} D^{T}>0 .
$$

which is equivalent to condition (16) with $Y=P^{-1} R P^{-1}$. By changing variable $X=$ $P^{-1}, Q<0$ in (29) can be expressed as

$$
\begin{aligned}
Q= & A X+X A^{T}-2 D D^{T}+\left(\frac{1}{\varepsilon}+\frac{1}{\mu}+\frac{1}{\rho}\right) E E^{T}+\frac{2}{\epsilon} I \\
& +Y+\varepsilon X F_{1}^{T} F_{1} X+2 \epsilon \gamma^{2} X X+\rho D D^{T} W^{-1} D D^{T} \\
< & 0 .
\end{aligned}
$$

With (30), it can be shown by Schur Complement that the condition (17) is equivalent to $Q<-\kappa_{1} I$, which further implies that

$$
\dot{V}(t) \leq-\kappa_{1}\|x(t)\|^{2}+\bar{\beta}(\|\omega(t)\|) .
$$

1) If $\omega(t) \equiv 0$, we have $\bar{\beta}(\|\omega(t)\|)=0$ by (31) and thus $\dot{V}(t)<0$. This implies that the uncertain closed-loop system (6) is asymptotically stable at the origin.

2) If $\omega(t) \in \mathcal{L}_{2}^{m}[0, \infty)$ is nonzero, integrating both sides of (33) obtains

$$
V(t) \leq V\left(t_{0}\right)-\kappa_{1} \int_{t_{0}}^{t}\|x(\tau)\|^{2} \mathrm{~d} \tau+\int_{t_{0}}^{t} \bar{\beta}(\|\omega(t)\|) \mathrm{d} \tau .
$$

Since $V(t)>0$, from (34) we have (11) with $\bar{\alpha}\left(t_{0}\right)=V\left(t_{0}\right) / \kappa_{1}$. This completes the poof.

Remark 2. It is worth mentioning that the decision variables of the LMI problem in (16) and (17) are $X$ and $Y$, while the scalars $\varepsilon, \mu, \rho$ and $\epsilon$ are positive constants introduced by Young inequality in stability analysis. For simplicity, $\varepsilon=\mu=\rho=$ $\epsilon=1$ can be set for solving the LMIs (16) and (17). However, these free parameters may provide additional design degrees of freedom and reduce the conservativeness of solutions.

Remark 3. It can be seen from (11) that the steady-state performance can be improved by increasing the parameter $\kappa_{1}$ and the disturbance attenuation can be therefore better achieved. However, a feasible solution $X>0$ of (17) may not exist for a very large $\kappa_{1}$. A compromise between performance and stability has to be balanced carefully in the control design.

Remark 4. For $\gamma=0$ (i.e., the linear case as studied in (Moon et al., 2001)), the right hand side of (19) is positive definite if and only if the pair $\left(A, F_{1}\right)$ is observable. For the case of unobservable pair $\left(A, F_{1}\right)$, it is much simpler to select $W$ since the right hand side of (19) with $\gamma=0$ is nonnegative definite. But for $\gamma \neq 0$, the right hand side of (19) is a positive definite matrix. In other words, a more stringent condition is needed for the selection of control gain due to the nonlinearity. One alternative way is that $W$ can be directly chosen as the inverse of the matrix in the right hand side of (19). 
Remark 5. The conditions (16) and (17) shown in Theorem 4.2 can be checked by standard LMI routines for a set of fixed values $R$ and $W^{-1}$. The iterative methods developed in (Yoon \& Lin, 2013) for linear systems may also be applied here.

Remark 6. It is also found that in spite of no explicit rule for us to judge the existence of a feasible solution in advance, if $A$ is Hurwitz or the Lipschitz constant $\gamma$ is small enough, it will become relatively easy to obtain a feasible solution $P>0$. In other words, for an open-loop unstable system with input delay, the existence of a solution to (16) and (17) cannot be guaranteed (Yoon \& Lin, 2013), and in addition, it would be harder to find a feasible solution if the influence of the nonlinearity is too big.

\section{Example}

Consider the system (6) with

$$
\begin{aligned}
& A=\left[\begin{array}{cc}
0 & -1 \\
1 & 0
\end{array}\right], B=\left[\begin{array}{cc}
1 & 0.5 \\
0 & 1.2
\end{array}\right], \Sigma(t)=\left[\begin{array}{cc}
\sin (t) & 0 \\
0 & \sin (2 t)
\end{array}\right], \\
& E=\left[\begin{array}{cc}
0.2 & 0 \\
0 & 0.2
\end{array}\right], F_{1}=\left[\begin{array}{cc}
0.1 & 0 \\
0 & 0.1
\end{array}\right], F_{2}=\left[\begin{array}{cc}
0.1 & 0 \\
0 & 0.1
\end{array}\right], \\
& \phi(x)=g\left[\begin{array}{c}
\sin \left(x_{1}\right) \\
0
\end{array}\right], B_{1}=\left[\begin{array}{l}
0 \\
1
\end{array}\right] .
\end{aligned}
$$

The linear nominal part of the system considered represents an oscillator, which covers a wide class of physical systems, like the electronic oscillator that produces a periodic, oscillating electronic signal. The Lipschitz nonlinearity may represent unmodeled or unknown dynamics. The initial condition $x(0)=[-2,1]^{T}$ is set in the simulation. The Lipschitz constant $\gamma=g=0.03$ and the delay $h=0.1$. With $\varepsilon=\mu=\rho=0.1, \kappa_{1}=$ $1, \epsilon=5$, a feasible solution of the feedback gain $K$ is found to be

$$
K=\left[\begin{array}{cc}
-0.9349 & 0.6384 \\
-0.0468 & -2.0017
\end{array}\right] \text {. }
$$

To validate the robust $\mathcal{L}_{2}$ disturbance attenuation performance, a normally distributed disturbance signal with zero mean and variance of 0.2 is added into (6) through $\omega(t)$. The simulation results shown in Figs. 1 and 2 demonstrate that the controller (4) with (10) (resp. asymptotically) stabilizes the input-delayed Lipschitz nonlinear system (resp. without) with external disturbances. The control inputs for the system in the presence of external disturbances are shown in Fig. 3. In addition, a better disturbance attenuation can be achieved with a larger $\kappa_{1}$, as shown in Fig. 4, which implies that the performance index (11) can be guaranteed by appropriately choosing $\kappa_{1}$. However, a larger overshoot is observed, compared with the state responses with a small $\kappa_{1}$. Thus, a tradeoff between the transient performance and the disturbance rejection performance should be carefully considered in practice. 


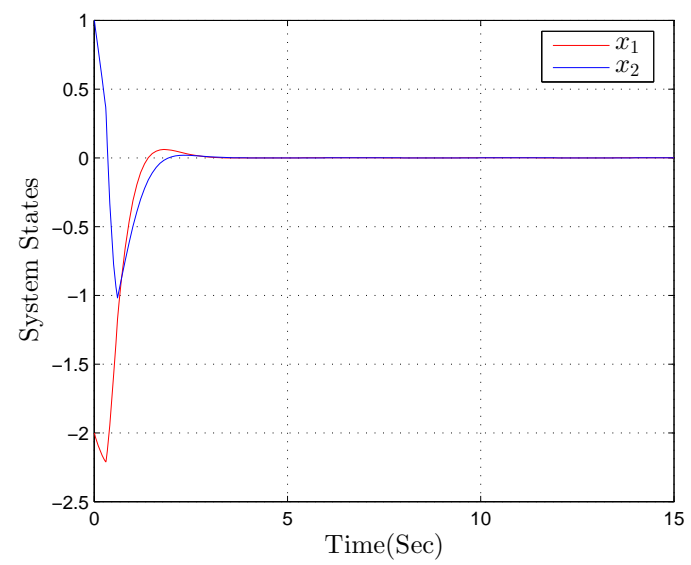

Figure 1. Closed-loop state profiles with $h=0.1$ and $\omega(t) \equiv 0$.

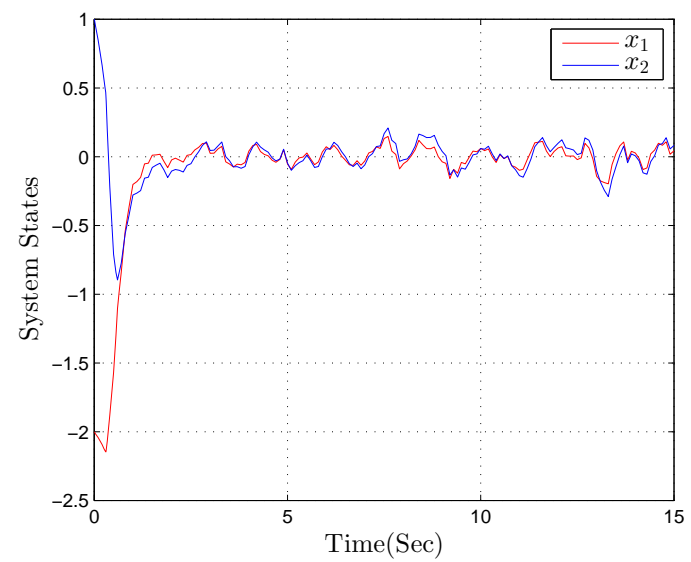

Figure 2. Closed-loop state profiles with $h=0.1$ and $\omega(t) \neq 0$.

\section{Conclusions}

In this paper, we have developed a robust predictor feedback control design for a class of uncertain Lipschitz nonlinear systems with input delay and external disturbances. The stability analysis is carried out in the framework of Lyapunov-Krasovskii functionals. Sufficient conditions in terms of two LMIs for the robust $\mathcal{L}_{2}$ disturbance attenuation are established with a set of iterative parameters. The effectiveness of the proposed designs has been demonstrated through a numerical example.

\section{References}

Anderson, B. \& Moore, J. (1989). Optimum control: Linear quadratic methods, New Jersey: Prentice-Hall.

Artstein, Z. (1982). Linear systems with delayed controls: a reduction. IEEE Transactions on Automatic Control, 27(4), 869-879.

Bekiaris-Liberis, N. \& Krstic, M. (2012). Compensation of time-varying input and state delays for nonlinear systems. Journal of Dynamic Systems-Transactions ASME, 134, 011009-1- 


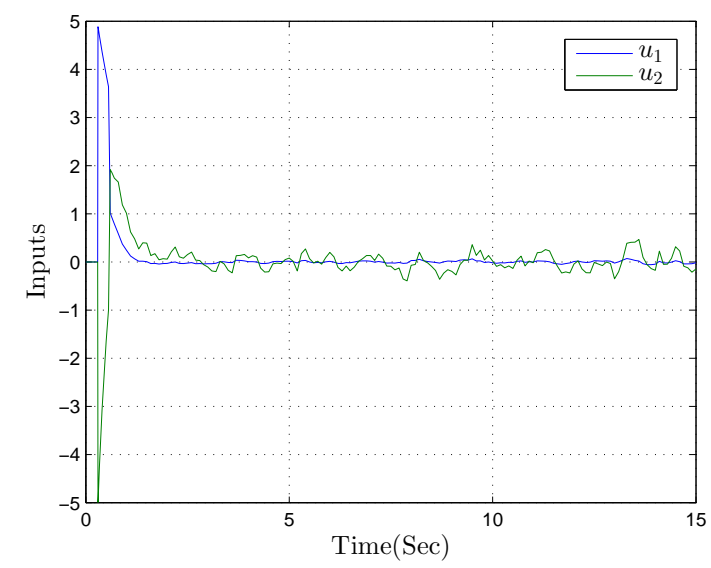

Figure 3. Input responses with $h=0.1$ and $\omega(t) \neq 0$.

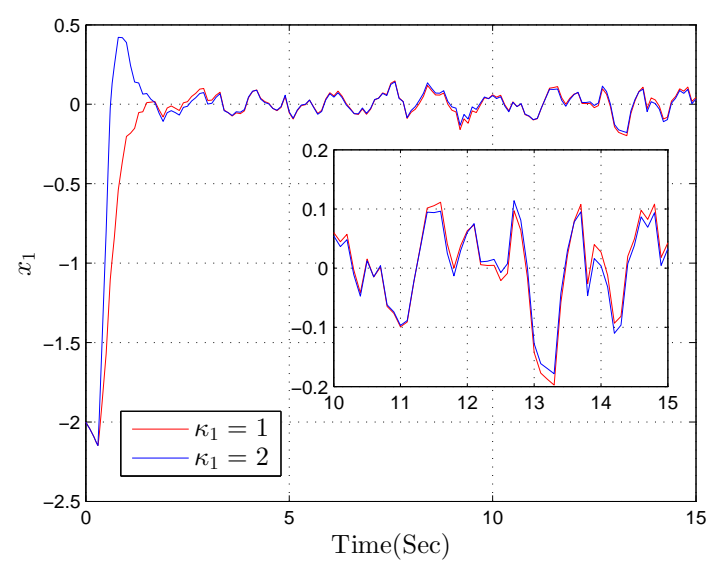

Figure 4. Closed-loop state profiles with different $\kappa_{1}$ 's.

011009-14.

Bekiaris-Liberis, N. \& Krstic, M. (2013). Nonlinear control under nonconstant delays. Philadelphia, PA: SIAM.

Choi, H.H. \& Chung, M.-H. (1995). Memoryless stabilization of uncertain dynamic systems with time-varying delayed state and control. Automatica, 31(9), 1349-1351.

$\mathrm{Gu}, \mathrm{K}$. (2010). An integral inequality in the stability problem of time-delay systems. Proceeding of 39th IEEE Conference Decision and Control, Sydney, Australia, pp. 2805-2810.

Gu, K., Kharitonov, V.L., \& Chen., J. (2003). Stability of time-delay systems. Boston, MA: Birkhäuser.

Hueschen, R.M. (2011). Development of the Transport Class Model (TCM) aircraft simulation from a sub-scale Generic Transport Model (GTM) simulation. NASA, Technical Memorandum 217169.

Krstic, M. (2010). Input delay compensation for forward complete and strict-feedforward nonlinear systems. IEEE Transactions on Automatic Control, 55(2), 287-303.

Kwon, W. \& Pearson, A. (1980). Feedback stabilization of linear systems with delayed control. IEEE Transactions on Automatic Control, 25(2), 266-269.

Kim, J.H., Jeung, E.T., \& Park, H.B. (1996). Robust control for parameter uncertain delay systems in state and control input. Automatica, 32(9), 1337-1339.

Lin, Z. \& Fang, H. (2007). On asymptotic stabilizability of linear systems with delayed input. 
IEEE Transactions on Automatic Control, 52(6), 998-1013.

Manitius, A. \& Olbrot, A. (1979). Finite spectrum assignment problem for systems with delays. IEEE Transactions on Automatic Control, $24(2), 541-552$.

Mazenc, F., Niculescu, S.I., \& Krstic, M. (2012). Lyapunov-Krasovskii functionals and applications to input delay compensation for linear-time-invariant systems. Automatica, 48(7), $1317-1323$.

Mondie S. \& Michiels, W. (2003). Finite spectrum assignment of unstable time-delay systems with a safe implementation. IEEE Transactions on Automatic Control, 48(12), 2207-2212.

Moon, Y.-S., Park, P., \& Kwon, W.-H. (2001) Robust stabilization of uncertain input-delayed systems using reduction method. Automatica, 37(2), 307-312.

Nguang, S.K. (2001). Comments on Robust stabilization of uncertain input-delay systems by sliding mode control with delay compensation. Automatica, 37(10), 1677-1677.

Pasillas-Lépine, W., Loría, A., \& Hoang, T.B. (2013). Preliminary results on output tracking control for restricted-feedback linearizable systems with input delay. Proceedings of 52nd IEEE Conference on Decision and Control, Florence, Italy, 2013, pp. 324-329.

Richard, J. (2003) Time-delay systems: An overview of some recent advances and open problems. Automatica, 39(10), 1667-1694.

Roh, Y.H. \& Oh, J.H. (1999). Robust stabilization of uncertain input-delay systems by sliding mode control with delay compensation. Automatica, 35(11), 1861-1865.

Smith, O. (1959). A controller to overcome dead time. ISA Journal, 6(2), 28-33.

Yoon, S.Y. \& Lin, Z. (2013). Truncated prediction feedback control for exponentially unstable linear systems with time-varying input delay. Systems \& Control Letters, 62(10), 837-844.

Yoon, S. Y., Anantachaisilp, P., \& Lin, Z. (2013). An LMI approach to the control of exponentially unstable systems with input time delay. Proceedings of 52nd IEEE Conference on Decision and Control, Florence, Italy, 2013, pp. 312-317.

Zhou, B., Lin, Z., \& Duan, G. (2010). Stabilization of linear systems with input delay and saturation - a parametric Lyapunov equation approach. International Journal of Robust and Nonlinear Control, 20(13), 1502-1519.

Zhou, B., Lin, Z., \& Duan, G. (2012). Truncated predictor feedback for linear systems with long time-varying input delays. Automatica, 48(10), 2387-2399.

Zuo, Z., Lin, Z., and Ding, Z. (2016). Truncated prediction output feedback control of a class of Lipschitz nonlinear systems with input delay. IEEE Transactions on Circuits and SystemsII: Brief Papers, 63(8), 788-792.

Zuo, Z., Lin, Z., and Ding, Z. (2016). Truncated predictor control of Lipschitz nonlinear systems with time-varying input delay. IEEE Transactions on Automatic Control, Published online, doi: 10.1109/TAC.2016.2635021. 